Online First version

\title{
Quasianalyticity in certain Banach function algebras
}

\author{
by
}

\author{
J. F. Feinstein and S. Morley (Nottingham)
}

\begin{abstract}
Let $X$ be a perfect, compact subset of the complex plane. We consider algebras of those functions on $X$ which satisfy a generalised notion of differentiability, which we call $\mathcal{F}$-differentiability. In particular, we investigate a notion of quasianalyticity under this new notion of differentiability and provide some sufficient conditions for certain algebras to be quasianalytic. We give an application of our results in which we construct an essential, natural uniform algebra $A$ on a locally connected, compact Hausdorff space $X$ such that $A$ admits no non-trivial Jensen measures yet is not regular. This construction improves an example of the first author (2001).
\end{abstract}

1. Introduction. Let $X$ be a perfect, compact subset of the complex plane $\mathbb{C}$. We consider those normed algebras consisting of complex-valued, continuously complex-differentiable functions on $X$, denoted $D^{(1)}(X)$. These algebras were introduced by Dales and Davie in [9] and further investigated, for example, in [2] and [10]. The algebra $D^{(1)}(X)$ need not be complete, and the completion of $D^{(1)}(X)$ need not be a Banach function algebra in general.

Bland and the first author [2] introduced $\mathcal{F}$-differentiation, which generalises the usual complex-differentiation, and considered normed algebras of $\mathcal{F}$-differentiable functions, denoted $D_{\mathcal{F}}^{(1)}(X)$. The algebra $D_{\mathcal{F}}^{(1)}(X)$ is complete and $D^{(1)}(X) \subseteq D_{\mathcal{F}}^{(1)}(X)$.

Dales and Davie [9] also considered those algebras of complex-valued functions which have continuous complex derivatives of all orders, and introduced the Dales-Davie algebras $D(X, M)$. They defined a notion of quasianalyticity for these algebras and gave sufficient conditions for $D(X, M)$ to be quasianalytic. (For the classical definition of quasianalytic collections of functions, see [27, Chapter 19].)

2010 Mathematics Subject Classification: Primary 46J10, 46J15; Secondary 46E25.

Key words and phrases: differentiable functions, Banach function algebra, uniform algebra, quasianalyticity, Jensen measures, Swiss cheeses.

Received 16 May 2016; revised 1 December 2016.

Published online 16 March 2017.

This paper contains work from the second author's PhD thesis. 
In this paper, we define a notion of quasianalyticity for infinitely $\mathcal{F}$-differentiable functions (defined later) and give a sufficient condition for this new notion of $\mathcal{F}$-quasianalyticity. In certain cases, this sufficient condition will improve that given by Dales and Davie [9]. We conclude the paper with the construction of a uniform algebra $A$ on a locally connected, compact Hausdorff space $X$ such that $A$ is essential and does not admit any non-trivial Jensen measures, yet is not regular. (The relevant definitions are given in Section 5.) This construction improves an example of the first author from [12].

2. Definitions and basic results. Throughout this paper we say compact plane set to mean a non-empty, compact subset of the complex plane $\mathbb{C}$. We denote the set of non-negative integers by $\mathbb{N}_{0}$, and the set of positive integers by $\mathbb{N}$. Let $X$ be a compact Hausdorff space. We denote the algebra (with pointwise operations) of all continuous, complex-valued functions on $X$ by $C(X)$. For $E \subseteq X$, we set

$$
|f|_{E}:=\sup _{x \in E}|f(x)| \quad(f \in C(X)) .
$$

With the norm $|\cdot|_{X}, C(X)$ is a commutative, unital Banach algebra. Let $S$ be a subset of $C(X)$. We say that $S$ separates the points of $X$ if, for each $x, y \in X$ with $x \neq y$, there exists $f \in S$ such that $f(x) \neq f(y)$.

Definition 2.1. Let $X$ be a compact Hausdorff space. A normed function algebra on $X$ is a normed algebra $(A,\|\cdot\|)$ such that $A$ is a subalgebra of $C(X), A$ contains all constant functions and separates the points of $X$, and, for each $f \in A,\|f\| \geq|f|_{X}$. A Banach function algebra on $X$ is a normed function algebra on $X$ which is complete. A uniform algebra on $X$ is a Banach function algebra on $X$ whose norm is $|\cdot|_{X}$.

Let $X$ be a compact Hausdorff space and let $A$ be a Banach function algebra on $X$. We say that $A$ is natural on $X$ if every character on $A$ is given by evaluation at some point of $X$.

We refer the reader to [8, Chapter 4] for further information on Banach function algebras and uniform algebras.

We are particularly interested in Banach function algebras consisting of continuous functions on a compact plane set which satisfy some notion of differentiability.

Definition 2.2. Let $X$ be a perfect compact plane set and let $f: X \rightarrow \mathbb{C}$ be a function. We say that $f$ is complex differentiable at $x \in X$ if the limit

$$
f^{\prime}(x):=\lim _{\substack{z \rightarrow x \\ z \in X}} \frac{f(z)-f(x)}{z-x}
$$


exists in $\mathbb{C}$. We say that $f$ is complex differentiable on $X$ if $f$ is complex differentiable at each point $x \in X$, and we call the function $f^{\prime}: X \rightarrow \mathbb{C}$ the derivative of $f$. We say that $f$ is continuously complex differentiable if $f^{\prime}$ is continuous.

In the remainder of this paper, we shall say differentiable and continuously differentiable to mean complex differentiable and continuously complex differentiable, respectively. We refer the reader to [7], for example, for results from complex analysis.

Let $X$ be a perfect compact plane set. We denote the algebra of all continuously differentiable functions on $X$ by $D^{(1)}(X)$. For each $n \in \mathbb{N}$, let $D^{(n)}(X)$ denote the algebra of all $n$-times continuously differentiable functions on $X$ (defined inductively). Let $D^{(\infty)}(X):=\bigcap_{n=1}^{\infty} D^{(n)}(X)$. Let $n \in \mathbb{N}$ and let $f \in D^{(n)}(X)$. We denote the $n$th derivative of $f$ by $f^{(n)}$, and we will often write $f^{(0)}$ for $f$.

We conclude this section with a discussion of paths in $\mathbb{C}$. For the remainder of this section, let $a, b \in \mathbb{R}$ with $a<b$.

Definition 2.3. A path in $\mathbb{C}$ is a continuous function $\gamma:[a, b] \rightarrow \mathbb{C}$. Let $\gamma:[a, b] \rightarrow \mathbb{C}$ be a path. The parameter interval of $\gamma$ is $[a, b]$. The endpoints of $\gamma$ are the points $\gamma(a)$ and $\gamma(b)$, which we denote by $\gamma^{-}$and $\gamma^{+}$, respectively. We denote by $\gamma^{*}$ the image $\gamma([a, b])$ of $\gamma$. A subpath of $\gamma$ is a path obtained by restricting $\gamma$ to a non-degenerate, closed subinterval of $[a, b]$. If $X$ is a subset of $\mathbb{C}$ then we say that $\gamma$ is a path in $X$ if $\gamma^{*} \subseteq X$.

Let $\gamma:[a, b] \rightarrow \mathbb{C}$ be a path in $\mathbb{C}$. We say that $\gamma$ is a Jordan path if $\gamma$ is an injective function. We denote the length of $\gamma$, as defined in [1, Chapter 6], by $\Lambda(\gamma)$, and we say that $\gamma$ is rectifiable if $\Lambda(\gamma)<\infty$, and non-rectifiable otherwise. We say that $\gamma$ is closed if $\gamma^{+}=\gamma^{-}$, and we say that $\gamma$ is a closed Jordan path if $\gamma$ is closed and $\gamma(s)=\gamma(t)$, where $s, t \in[a, b]$, implies that either $s=t$ or $s=a$ and $t=b$. We say that $\gamma$ is admissible if $\gamma$ is rectifiable and has no constant subpaths. The reverse of $\gamma$ is the path $-\gamma:[-b,-a] \rightarrow \gamma^{*}$ given by $-\gamma(t)=\gamma(-t)$. It is standard that

$$
\int_{-\gamma} f(z) d z=-\int_{\gamma} f(z) d z \quad\left(f \in C\left(\gamma^{*}\right)\right) .
$$

Now suppose that $\gamma$ is non-constant and rectifiable. We define the path length parametrisation $\gamma^{\mathrm{pl}}:[0, \Lambda(\gamma)] \rightarrow \mathbb{C}$ of $\gamma$ to be the unique path satisfying $\gamma^{\mathrm{pl}}(\Lambda(\gamma \mid[a, t]))=\gamma(t)(t \in[a, b])$; see, for example, [11, pp. 109-110] for details. We define the normalised path length parametrisation of $\gamma$, $\gamma^{\text {no }}:[0,1] \rightarrow \mathbb{C}$, to be the path such that $\gamma^{\text {no }}(t)=\gamma^{\mathrm{pl}}(t \Lambda(\gamma))$ for each $t \in[0,1]$. It is clear that $\gamma^{\mathrm{pl}}$ and $\gamma^{\text {no }}$ are necessarily admissible paths and $\left(\gamma^{\mathrm{pl}}\right)^{*}=\left(\gamma^{\mathrm{no}}\right)^{*}=\gamma^{*}$. It is not hard to show, using [11, Theorem 2.4.18], 
that

$$
\int_{\gamma} f(z) d z=\int_{\gamma^{\mathrm{pl}}} f(z) d z=\int_{\gamma^{\text {no }}} f(z) d z
$$

for all $f \in C\left(\gamma^{*}\right)$. We shall use this fact implicitly throughout.

Definition 2.4. Let $X$ be a perfect compact plane set and let $\mathcal{F}$ be a collection of paths in $X$. Define $\mathcal{F}^{*}:=\left\{\gamma^{*}: \gamma \in \mathcal{F}\right\}$. We say that $\mathcal{F}$ is effective if $\bigcup_{\gamma \in \mathcal{F}} \gamma^{*}$ is dense in $X$, each path $\gamma \in \mathcal{F}$ is admissible, and each subpath of a path in $\mathcal{F}$ belongs to $\mathcal{F}$.

Let $X$ be a compact plane set. We say that $X$ is semirectifiable if the set of all Jordan paths in $X$ is an effective collection of paths in $X$. We say that $X$ is rectifiably connected if, for each $x, y \in X$, there exists a rectifiable path $\gamma \in \mathcal{F}$ such that $\gamma^{-}=x$ and $\gamma^{+}=y$. We say that $X$ is uniformly regular if there exists a constant $C>0$ such that for all $x, y \in X$ there exists a rectifiable path $\gamma$ in $X$ with $\gamma^{-}=x$ and $\gamma^{+}=y$ such that $\Lambda(\gamma) \leq C|x-y|$. We say that $X$ is pointwise regular if for each $x \in X$ there exists a constant $C_{x}>0$ such that, for each $y \in X$, there exists a path $\gamma$ in $X$ with $\gamma^{-}=x$ and $\gamma^{+}=y$ such that $\Lambda(\gamma) \leq C_{x}|x-y|$. Note that each of the above conditions on $X$ implies that $X$ is perfect.

3. $\mathcal{F}$-derivatives. In this section we discuss algebras of $\mathcal{F}$-differentiable functions as investigated in [2] and [10].

Definition 3.1. Let $X$ be a perfect compact plane set, let $\mathcal{F}$ be a collection of rectifiable paths in $X$, and let $f \in C(X)$. A function $g \in C(X)$ is an $\mathcal{F}$-derivative for $f$ if, for each $\gamma \in \mathcal{F}$, we have

$$
\int_{\gamma} g(z) d z=f\left(\gamma^{+}\right)-f\left(\gamma^{-}\right) .
$$

If $f$ has an $\mathcal{F}$-derivative on $X$ then we say that $f$ is $\mathcal{F}$-differentiable on $X$.

The following proposition is a list of the elementary properties of $\mathcal{F}$-differentiable functions. Details can be found in [2] and [10].

Proposition 3.2. Let $X$ be a semirectifiable compact plane set and let $\mathcal{F}$ be an effective collection of paths in $X$.

(a) Let $f, g, h \in C(X)$ be such that $g$ and $h$ are $\mathcal{F}$-derivatives for $f$. Then $g=h$.

(b) Let $f \in D^{(1)}(X)$. Then the usual complex derivative of $f$ on $X, f^{\prime}$, is an $\mathcal{F}$-derivative for $f$.

(c) Let $f_{1}, f_{2}, g_{1}, g_{2} \in C(X)$ be such that $g_{1}$ is an $\mathcal{F}$-derivative for $f_{1}$ and $g_{2}$ is an $\mathcal{F}$-derivative for $f_{2}$. Then $f_{1} g_{2}+g_{1} f_{2}$ is an $\mathcal{F}$-derivative for $f_{1} f_{2}$. 
(d) Let $f_{1}, f_{2}, g_{1}, g_{2} \in C(X)$ and $\alpha, \beta \in \mathbb{C}$ be such that $g_{1}$ is an $\mathcal{F}$-derivative for $f_{1}$ and $g_{2}$ is an $\mathcal{F}$-derivative for $f_{2}$. Then $\alpha g_{1}+\beta g_{2}$ is an $\mathcal{F}$-derivative for $\alpha f_{1}+\beta f_{2}$.

Let $X$ be a semirectifiable compact plane set, and let $\mathcal{F}$ be an effective collection of paths in $X$. By (a) above, $\mathcal{F}$-derivatives are unique. So, in this setting, we write $f^{[1]}$ for the unique $\mathcal{F}$-derivative of an $\mathcal{F}$-differentiable function. This will be the case considered throughout the remainder of this paper. We will often write $f^{[0]}$ for $f$. We write $D_{\mathcal{F}}^{(1)}(X)$ for the algebra of all $\mathcal{F}$-differentiable functions on $X$. With the norm $\|f\|_{\mathcal{F}, 1}:=|f|_{X}+\left|f^{[1]}\right|_{X}$ $\left(f \in D_{\mathcal{F}}^{(1)}(X)\right)$, the algebra $D_{\mathcal{F}}^{(1)}(X)$ is a Banach function algebra on $X$ [10, Theorem 5.6].

For each integer $n \geq 2$, we define (inductively) the algebra

$$
D_{\mathcal{F}}^{(n)}(X):=\left\{f \in D_{\mathcal{F}}^{(1)}(X): f^{[1]} \in D_{\mathcal{F}}^{(n-1)}(X)\right\},
$$

and, for each $f \in D_{\mathcal{F}}^{(n)}(X)$, we write $f^{[n]}$ for the $n$th $\mathcal{F}$-derivative for $f$. Note that, for each $n \in \mathbb{N}, D_{\mathcal{F}}^{(n)}(X)$ is a Banach function algebra on $X$ (see [2]) when given the norm

$$
\|f\|_{\mathcal{F}, n}:=\sum_{k=0}^{n} \frac{\left|f^{[k]}\right|_{X}}{k !} \quad\left(f \in D_{\mathcal{F}}^{(n)}(X)\right) .
$$

In addition, we define the algebra $D_{\mathcal{F}}^{(\infty)}(X)$ of all functions which have $\mathcal{F}$-derivatives of all orders, that is, $D_{\mathcal{F}}^{(\infty)}(X)=\bigcap_{n=1}^{\infty} D_{\mathcal{F}}^{(n)}(X)$. It is easy to see that $D^{(n)}(X) \subseteq D_{\mathcal{F}}^{(n)}(X)$ for each $n \in \mathbb{N}$, and $D^{(\infty)}(X) \subseteq D_{\mathcal{F}}^{(\infty)}(X)$.

DEFInition 3.3. Let $M=\left(M_{n}\right)_{n=0}^{\infty}$ be a sequence of positive real numbers. We say that $M$ is an algebra sequence if $M_{0}=1$ and, for each $j, k \in \mathbb{N}_{0}$, we have

$$
\left(\begin{array}{c}
j+k \\
k
\end{array}\right) \leq \frac{M_{j+k}}{M_{j} M_{k}}
$$

We say that $M$ is log-convex if, for each $k \in \mathbb{N}$, we have $M_{k}^{2} \leq M_{k-1} M_{k+1}$.

We now describe a class of algebras of infinitely $\mathcal{F}$-differentiable functions analogous to Dales-Davie algebras as introduced in [2] (see also [4]).

Definition 3.4. Let $X$ be a semirectifiable, compact plane set and let $\mathcal{F}$ be an effective collection of paths in $X$. Let $M=\left(M_{n}\right)_{n=0}^{\infty}$ be an algebra sequence. We define the normed algebra

$$
D_{\mathcal{F}}(X, M):=\left\{f \in D_{\mathcal{F}}^{(\infty)}(X): \sum_{j=0}^{\infty} \frac{\left|f^{[j]}\right|_{X}}{M_{j}}<\infty\right\}
$$


with pointwise operations and the norm

$$
\|f\|:=\sum_{j=0}^{\infty} \frac{\left|f^{[j]}\right|_{X}}{M_{j}} \quad\left(f \in D_{\mathcal{F}}(X, M)\right) .
$$

Unlike the Dales-Davie algebras discussed in [9], the algebras $D_{\mathcal{F}}(X, M)$ described above are always complete.

4. $\mathcal{F}$-quasianalyticity. In this section, we discuss an $\mathcal{F}$-differentiability version of quasianalyticity, and give a sufficient condition for a subalgebra of $D_{\mathcal{F}}^{(\infty)}(X)$ to satisfy this new notion of quasianalyticity.

We now introduce the following notion of $\mathcal{F}$-quasianalyticity.

Definition 4.1. Let $X$ be a semirectifiable compact plane set and let $\mathcal{F}$ be an effective collection of paths in $X$. Let $A$ be a subalgebra of $D_{\mathcal{F}}^{(\infty)}(X)$. Then $A$ is $\mathcal{F}$-quasianalytic if, for each $\gamma \in \mathcal{F}$ and $z_{0} \in \gamma^{*}$, the conditions

$$
f \in A \quad \text { and } \quad f^{[k]}\left(z_{0}\right)=0 \quad(k=0,1, \ldots)
$$

together imply that $f(z)=0$ for all $z \in \gamma^{*}$.

Let $X$ be a semirectifiable compact plane set and let $\mathcal{F}$ be an effective collection of paths in $X$. We aim to give some sufficient conditions for $\mathcal{F}$-quasianalyticity of the algebras $D_{\mathcal{F}}(X, M)$. Our method will follow the proof in [6] of the traditional Denjoy-Carleman theorem.

For the remainder of the section we fix an admissible path $\Gamma$. We also fix $\mathcal{F}$ to be the collection of all subpaths and reverses of subpaths of $\Gamma$. Let $M=\left(M_{n}\right)_{n=0}^{\infty}$ be a sequence of positive real numbers satisfying

$$
\sum_{n=1}^{\infty} M_{n}^{-1 / n}=\infty
$$

We write $d s$ for integrals with respect to the path length measure. Let $D$ be the algebra $D_{\mathcal{F}}^{(\infty)}\left(\Gamma^{*}\right)$ and, for each $n \in \mathbb{N}$, set $D_{n}:=D_{\mathcal{F}}^{(n)}\left(\Gamma^{*}\right)$.

We will require the following lemmas. The first is a summary of the properties of the log-convex minorant of a sequence of positive real numbers. We refer the reader to [25, Chapter IV] and [26, Chapter 1] for details and properties of the log-convex minorants. The properties listed below are from [6].

LEMma 4.2. Let $\left(M_{n}\right)_{n=0}^{\infty}$ be a sequence of positive real numbers such that $\liminf _{n \rightarrow \infty} M_{n}^{1 / n}=\infty$. Then there exists a log-convex sequence $\left(M_{n}^{\mathrm{c}}\right)_{n=0}^{\infty}$ and a strictly increasing sequence $\left(n_{j}\right)_{j=0}^{\infty}$ of integers with $n_{0}=0$ such that:

(a) $M_{k}^{c} \leq M_{k}$ for all $k \in \mathbb{N}_{0}$;

(b) $M_{n_{k}}^{\mathrm{c}}=M_{n_{k}}$ for all $k \in \mathbb{N}_{0}$;

(c) for each $k \in \mathbb{N}_{0}$, we have $M_{j}^{\mathrm{c}} / M_{j+1}^{\mathrm{c}}=M_{n_{k}}^{\mathrm{c}} / M_{n_{k}+1}^{\mathrm{c}}$ for all $j \in \mathbb{N}_{0}$ with $n_{k} \leq j<n_{k+1}$. 
It is easy to see that if a sequence $M=\left(M_{n}\right)_{n=0}^{\infty}$ of positive real numbers satisfies (4.1) then the log-convex minorant $M^{\mathrm{c}}=\left(M_{n}^{\mathrm{c}}\right)_{n=0}^{\infty}$, as given by Lemma 4.2 satisfies (4.1). Moreover, if $M^{\mathrm{c}}$ satisfies (4.1) then $M^{\mathrm{c}}$ satisfies $\sum_{n=0}^{\infty} M_{n}^{\mathrm{c}} / M_{n+1}^{\mathrm{c}}=\infty$; see [25, Chapter IV] for details.

The second lemma is standard; see, for example, [7, Proposition 1.17].

Lemma 4.3. Let $\gamma \in \mathcal{F}$ and let $f \in D_{1}$. Then

$$
\left|f\left(\gamma^{+}\right)\right| \leq\left|f\left(\gamma^{-}\right)\right|+\Lambda(\gamma)\left|f^{[1]}\right|_{\gamma^{*}}
$$

Our next lemma is an $\mathcal{F}$-differentiability analogue of [6, Lemma 2].

Lemma 4.4. Let $\gamma \in \mathcal{F}$, let $M>0$, let $m \in \mathbb{N}$, and let $\sigma=\gamma^{\mathrm{pl}}$. Let $s_{0}, \ldots, s_{m-1} \in[0, \Lambda(\sigma)]$ be such that $0 \leq s_{0}<s_{1}<\cdots<s_{m-1}<\Lambda(\sigma)$. Let $g \in D_{m}$ and suppose that $\left|g^{[m]}(\sigma(t))\right| \leq M$ for all $t \in\left[s_{0}, \Lambda(\gamma)\right]$. Then, for all $s \in\left(s_{m-1}, \Lambda(\gamma)\right]$, we have

$$
|g(\sigma(s))| \leq \sum_{p=0}^{m-1} \frac{\left|g^{[p]}\left(\sigma\left(s_{m-p-1}\right)\right)\right|\left(s-s_{m-p-1}\right)^{p}}{p !}+\frac{M}{m !}\left(s-s_{0}\right)^{m} .
$$

Proof. Note that, for each $a, b \in[0, \Lambda(\gamma)]$ with $a<b, \Lambda(\sigma \mid[a, b])=b-a$. We prove the result by induction on $m$. If $m=1$ then, by Lemma 4.3 applied to $\sigma \mid\left[s_{0}, s\right]$, we have

$$
|g(\sigma(s))| \leq\left|g\left(\sigma\left(s_{0}\right)\right)\right|+M \Lambda\left(\sigma \mid\left[s_{0}, s\right]\right)=\left|g\left(\sigma\left(s_{0}\right)\right)\right|+M\left(s-s_{0}\right) .
$$

Let $m>1$ be an integer and assume that the result holds for $m-1$. Since $g^{[1]} \in D_{m-1}$, it follows that, for each $s \in\left[s_{m-2}, \Lambda(\gamma)\right]$, we have $\left|g^{[1]}(\sigma(s))\right| \leq \sum_{p=0}^{m-2} \frac{\left|g^{[p+1]}\left(\sigma\left(s_{m-p-2}\right)\right)\right|\left(s-s_{m-p-2}\right)^{p}}{p !}+\frac{M}{(m-1) !}\left(s-s_{0}\right)^{m-1}$.

Fix $t \in\left(s_{m-1}, \Lambda(\gamma)\right]$. For each $j \in\{1, \ldots, m-1\}$ let $\sigma_{j}:=\sigma \mid\left[s_{j}, t\right]$. Then

$$
\begin{aligned}
\int_{\sigma_{m-1}}\left|g^{[1]}(\sigma(s))\right| d s \leq & \int_{\sigma_{m-1}} \sum_{p=0}^{m-2} \frac{\left|g^{[p+1]}\left(\sigma\left(s_{m-p-2}\right)\right)\right|\left(s-s_{m-p-2}\right)^{p}}{p !} d s \\
& +\int_{\sigma_{m-1}} \frac{M}{(m-1) !}\left(s-s_{0}\right)^{m-1} d s .
\end{aligned}
$$

For each $j \in\{1, \ldots, m-2\}$, we have

$$
\int_{\sigma_{m-1}}\left(s-s_{m-j-1}\right)^{j-1} d s \leq \int_{\sigma_{m-j-1}}\left(s-s_{m-j-1}\right)^{j-1} d s=\frac{\left(t-s_{m-j-1}\right)^{j}}{j},
$$


and, by combining 4.3 and 4.2, we obtain

$$
\int_{\sigma_{m-1}}\left|g^{[1]}(\sigma(s))\right| d s \leq \sum_{p=1}^{m-1} \frac{\left|g^{[p]}\left(\sigma\left(s_{m-p-1}\right)\right)\right|\left(t-s_{m-p-1}\right)^{p}}{p !}+\frac{M}{m !}\left(t-s_{0}\right)^{m} .
$$

But now

$$
|g(\sigma(t))| \leq\left|g\left(\sigma\left(s_{m-1}\right)\right)\right|+\int_{\sigma_{m-1}}\left|g^{[1]}(\sigma(s))\right| d s,
$$

and combining this with 4.4, we obtain the desired result.

We now check an easy special case of our result.

Lemma 4.5. Let $M=\left(M_{n}\right)_{n=0}^{\infty}$ be a sequence of positive real numbers such that $\liminf _{n \rightarrow \infty} M_{n}^{1 / n}<\infty$. Let $z_{0} \in \Gamma^{*}$, and let $f \in D$ with $\left|f^{[k]}\right|_{\Gamma^{*}} \leq M_{k}$ and $f^{[k]}\left(z_{0}\right)=0$ for all $k \in \mathbb{N}_{0}$. Then $f(z)=0$ for all $z \in \Gamma^{*}$.

Proof. Fix $z \in \Gamma^{*}$. Let $\gamma \in \mathcal{F}$ be such that $\gamma^{-}=z_{0}, \gamma^{+}=z$, and $\gamma=\gamma^{\mathrm{pl}}$. We first claim that, for each $k, n \in \mathbb{N}_{0}$ with $k \leq n$, we have

$$
\left|f^{[k]}(z)\right| \leq M_{n} \frac{s^{n-k}}{(n-k) !}
$$

where $s=\Lambda(\gamma)$.

Fix $n \in \mathbb{N}_{0}$. We prove our claim by backwards induction on $k \leq n$. The case where $k=n$ is trivial. Now suppose that the result holds for some positive integer $k \leq n$. Let $z \in \gamma^{*}$. We have

$$
\left|f^{[k-1]}(z)\right| \leq\left|f^{[k-1]}\left(\gamma^{+}\right)\right|+\int_{\gamma}\left|f^{[k]}\right|_{\gamma^{*}} d s=\int_{\gamma}\left|f^{[k]}\right|_{\gamma^{*}} d s
$$

and applying the claim to $\left|f^{[k]}(\zeta)\right|$, we obtain

$$
\left|f^{[k-1]}(z)\right| \leq \int_{\gamma} \frac{M_{n} s^{n-k}}{(n-k) !} d s=\frac{M_{n} s^{n-k+1}}{(n-k+1) !} .
$$

This proves the claim.

We now see that $|f(z)| \leq M_{n} s^{n} / n$ ! for all $n \in \mathbb{N}_{0}$ and all $z \in \gamma^{*}$. Since $\liminf _{n \rightarrow \infty} M_{n}^{1 / n}<\infty$, there exists $R>0$ such that $M_{n}<R^{n}$ for infinitely many $n \in \mathbb{N}$. Let $\left(n_{k}\right)_{k=1}^{\infty}$ be a strictly increasing sequence in $\mathbb{N}$ such that $M_{n_{k}}<R^{n_{k}}$ for all $k \in \mathbb{N}$. Then, for each $z \in \gamma^{*}$, we have

$$
|f(z)| \leq \frac{M_{n_{k}} s^{n_{k}}}{n_{k} !}<\frac{(R s)^{n_{k}}}{n_{k} !} \rightarrow 0
$$

as $k \rightarrow \infty$. This holds for all $z \in \Gamma^{*}$, so the result follows.

Let $0<\alpha<1$. As in [6], we define $B_{j, k}^{(\alpha)}$, for $j, k \in \mathbb{N}_{0}$ with $k \geq j \geq 0$, as follows. For each $j \in \mathbb{N}_{0}$, let $B_{0, j}^{(\alpha)}=0$, and for each $j \in \mathbb{N}$, let $B_{j, j}^{(\alpha)}=1$. For 
each $j, k \in \mathbb{N}_{0}$ with $k>j$, define $B_{j+1, k+1}^{(\alpha)}$ inductively by setting

$$
B_{j+1, k+1}^{(\alpha)}=B_{j, k+1}^{(\alpha)}+\alpha B_{j+1, k}^{(\alpha)} .
$$

Our main tool in the proof of the main theorem is the following lemma, which can be distilled from the proof of [6, Lemma 1] and Stirling's approximation. We omit the proof.

LEMmA 4.6. Let $\alpha \in\left(0,1 /\left(2 e^{2}\right)\right)$. Then there exists a constant $K>0$ such that $B_{j, k}^{(\alpha)} \leq K \alpha<1 / 2$ for all $j, k \in \mathbb{N}$ with $j<k$. Moreover, for each $n \in \mathbb{N}$, we have

$$
\sum_{j=0}^{n-1} \frac{((j+1) \alpha)^{j}}{j !}+2 \frac{(n \alpha)^{n}}{n !}<2 .
$$

Note that if $\alpha \in\left(0,1 /\left(2 e^{2}\right)\right)$ then

$$
B_{j+1, k+1}^{(\alpha)} \geq B_{j, k+1}^{(\alpha)}
$$

for all $j, k \in \mathbb{N}_{0}$ with $k \geq j$.

We now state and prove our main result. The proof is essentially the one used in [6], adapted for $\mathcal{F}$-differentiation, and including additional details for the convenience of the reader.

TheOREM 4.7. Let $\Gamma:[a, b] \rightarrow \mathbb{C}$ be an admissible path and let $\mathcal{F}$ denote the collection of all subpaths and reverses of subpaths of $\Gamma$. Let $\left(M_{n}\right)_{n=0}^{\infty}$ be a sequence of positive real numbers such that $\sum_{n=1}^{\infty} M_{n}^{-1 / n}=\infty$. Suppose that $f \in D_{\mathcal{F}}^{(\infty)}\left(\Gamma^{*}\right)$ and $z_{0} \in \Gamma^{*}$ satisfy

$$
\left|f^{[k]}\right|_{\Gamma^{*}} \leq M_{k} \quad \text { and } \quad f^{[k]}\left(z_{0}\right)=0
$$

for all $k \in \mathbb{N}_{0}$. Then $f(z)=0$ for all $z \in \Gamma^{*}$.

Proof. If $\lim \inf _{n \rightarrow \infty} M_{n}^{1 / n}<\infty$ then the result follows from Lemma 4.5 . so suppose that $\liminf _{n \rightarrow \infty} M_{n}^{1 / n}=\infty$. By Lemma 4.2 , there exists a logconvex sequence $\left(M_{n}^{\mathrm{c}}\right)_{n=0}^{\infty}$ of positive real numbers and a strictly increasing sequence $\left(n_{j}\right)_{j=0}^{\infty}$ with $n_{0}=0$ such that $(\mathrm{a})-(\mathrm{c})$ of the lemma are satisfied. By the comments following the lemma, we have

$$
\sum_{n=0}^{\infty} \frac{M_{n}^{\mathrm{c}}}{M_{n+1}^{\mathrm{c}}}=\infty
$$

Let $z \in \Gamma^{*}$ and let $\gamma \in \mathcal{F}$ be such that $\gamma^{-}=z_{0}, \gamma^{+}=z$ and $\sigma=\gamma^{\mathrm{pl}}$.

Fix $n \in \mathbb{N}$ that is an element in the sequence $\left(n_{p}\right)_{p=0}^{\infty}$ and satisfies the condition $\sum_{j=0}^{n} M_{j}^{\mathrm{c}} / M_{j+1}^{\mathrm{c}}>2 e^{2} \Lambda(\sigma)$. Let $\alpha:=\Lambda(\sigma) /\left(\sum_{j=0}^{n} M_{j}^{\mathrm{c}} / M_{j+1}^{\mathrm{c}}\right)$. Then $\alpha \in\left(0,1 /\left(2 e^{2}\right)\right)$, and so, by Lemma 4.6. $B_{u, v}^{(\alpha)}<1 / 2$ for all $u, v \in \mathbb{N}$ with $v>u>0$. 
Define the points $0=x_{0}<x_{1}<\cdots<x_{n} \leq \Lambda(\sigma)$ such that

$$
x_{j}-x_{j-1}=\alpha M_{n-j}^{\mathrm{c}} / M_{n-j+1}^{\mathrm{c}}, \quad j=1, \ldots, n .
$$

For each $j \in\{0, \ldots, n\}$ we claim that, for each $k \in \mathbb{N}_{0}$ with $k \leq n-j+1$,

$$
\left|f^{[k]}(\sigma(t))\right| \leq B_{j, n-k+1}^{(\alpha)} M_{k}^{\mathrm{c}} \quad\left(t \in\left[0, x_{j}\right]\right) .
$$

The proof of the claim is by induction on $j$. Since $f^{[k]}\left(\sigma^{-}\right)=0$ for each $k \in \mathbb{N}_{0},\left(*_{k}^{0}\right)$ holds for all $k \in \mathbb{N}_{0}$ with $k \leq n+1$.

Fix $j \in\{1, \ldots, n\}$. Assume now that $\left(*_{k^{\prime}}^{j^{\prime}}\right)$ holds for all $j^{\prime}, k^{\prime} \in \mathbb{N}_{0}$ with $j^{\prime}<j$ and $k^{\prime} \leq n-j^{\prime}+1$. Set $i:=n-j+1$. We prove $\left(*_{k}^{j}\right)$ for each $k \in \mathbb{N}_{0}$ with $k \leq i$ by backwards induction on $k$. We first check the base case. Suppose that $k=i$. If $i=n_{r}$ for some $r \in \mathbb{N}_{0}$, then $B_{j, j}^{(\alpha)}=1$ and $\left|f^{[i]}(\sigma(t))\right| \leq M_{i}=M_{i}^{\text {c }}$ for all $t \in\left[0, x_{j}\right]$ by (4.7), and so $\left(*_{k}^{j}\right)$ holds.

Otherwise, $i \neq n_{r}$ for all $r \in \mathbb{N}_{0}$, in which case there exists $r \in \mathbb{N}_{0}$ such that $n_{r}<i<n_{r+1} \leq n$. For each $s \in\left[0, x_{j-1}\right]$, by $\left(*_{i}^{j-1}\right)$ and (4.6), we have

$$
\left|f^{[i]}(\sigma(s))\right| \leq B_{j-1, j}^{(\alpha)} M_{i}^{\mathrm{c}} \leq B_{j, j}^{(\alpha)} M_{i}^{\mathrm{c}}=M_{i}^{\mathrm{c}},
$$

and so it remains to show that $\left|f^{[i]}(\sigma(s))\right| \leq M_{i}^{\mathrm{c}}$ for all $s \in\left[x_{j-1}, x_{j}\right]$.

As in [6], let $m:=n_{r+1}-i$ and let $R:=M_{n_{r}}^{\mathrm{c}} / M_{n_{r}+1}^{\mathrm{c}}$. Note that

$$
j-m=n-n_{r+1}+1 \geq 1
$$

and, for each $p \in\{0, \ldots, m\}$, we have $M_{i}^{\mathrm{c}}=M_{i+p}^{\mathrm{c}} R^{p}$. Also, for each $\ell \in \mathbb{N}$ with $n_{r} \leq \ell<n_{r+1}$, we have $M_{\ell}^{\mathrm{c}} / M_{\ell+1}^{\mathrm{c}}=R$, and so $x_{j-p}-x_{j-p-1}=\alpha R$ for each $p \in\{1, \ldots, m\}$. In particular, $M_{i}^{\mathrm{c}}=M_{n_{r+1}}^{\mathrm{c}} R^{m}$. For each $s \in\left[x_{j-1}, x_{j}\right]$, by Lemma 4.4 (applied with $M=M_{n_{r+1}}^{\mathrm{c}}=M_{n_{r+1}}, g=f^{[i]}$ and points $\left.s_{0}=x_{j-m}, \ldots, s_{m-1}=x_{j-1}\right)$, we have

$$
\begin{aligned}
\left|f^{[i]}(\sigma(s))\right| & \leq \sum_{p=0}^{m-1} \frac{\left|f^{[i+p]}\left(\sigma\left(x_{j-p-1}\right)\right)\right|\left(s-x_{j-p-1}\right)^{p}}{p !}+\frac{M_{n_{r+1}}^{\mathrm{c}}}{m !}\left(s-x_{j-m}\right)^{m} \\
& \leq \sum_{p=0}^{m-1} \frac{\left|f^{[i+p]}\left(\sigma\left(x_{j-p-1}\right)\right)\right|((p+1) \alpha R)^{p}}{p !}+\frac{M_{n_{r+1}}^{\mathrm{c}}}{m !}(m \alpha R)^{m},
\end{aligned}
$$

and by applying $\left(*_{i+p}^{j-p-1}\right)$ for each $0 \leq p \leq m-1$, we obtain

$$
\begin{aligned}
\left|f^{[i]}(\sigma(s))\right| & \leq \sum_{p=0}^{m-1} \frac{B_{j-1-p, n-(i+p)+1}^{(\alpha)} M_{i+p}^{\mathrm{c}}((p+1) \alpha R)^{p}}{p !}+\frac{M_{n_{r+1}}^{\mathrm{c}}(m \alpha R)^{m}}{m !} \\
& =M_{i}^{\mathrm{c}}\left(\sum_{p=0}^{m-1} \frac{B_{j-1-p, j-p}^{(\alpha)}((p+1) \alpha)^{p}}{p !}+\frac{(m \alpha)^{m}}{m !}\right) .
\end{aligned}
$$


Since $B_{u, v}^{(\alpha)}<1 / 2$ for all $u, v \in \mathbb{N}_{0}$ with $v>u>0$, we have

$$
\left|f^{[i]}(\sigma(s))\right| \leq \frac{M_{i}^{\mathrm{c}}}{2}\left(\sum_{p=0}^{m-1} \frac{((p+1) \alpha)^{p}}{p !}+2 \frac{(m \alpha)^{m}}{m !}\right),
$$

and so, by Lemma 4.6 , we obtain $\left|f^{[i]}(\sigma(s))\right|<M_{i}^{\mathrm{c}}$. This concludes the proof of the base case $k=i$.

Now let $k \in \mathbb{N}_{0}$ with $k \leq i-1$ and assume that $\left(*_{k+1}^{j}\right)$ holds, i.e.,

$$
\left|f^{[k+1]}(\sigma(t))\right| \leq B_{j, n-(k+1)+1}^{(\alpha)} M_{k+1}^{\mathrm{c}}
$$

for all $t \in\left[0, x_{j}\right]$. Let $s \in\left[0, x_{j}\right]$. If $s \in\left[0, x_{j-1}\right]$ then, by $\left(*_{k}^{j-1}\right)$ and 4.6 , we have

$$
\left|f^{[k]}(\sigma(s))\right| \leq B_{j-1, n-k+1}^{(\alpha)} M_{k}^{\mathrm{c}} \leq B_{j, n-k+1}^{(\alpha)} M_{k}^{\mathrm{c}} .
$$

Thus we may assume that $s \in\left[x_{j-1}, x_{j}\right]$. By Lemma 4.3 . $\left|f^{[k]}(\sigma(s))\right| \leq\left|f^{[k]}\left(\sigma\left(x_{j-1}\right)\right)\right|+\left(x_{j}-x_{j-1}\right) \sup \left\{\left|f^{[k+1]}(\sigma(t))\right|: t \in\left[x_{j-1}, x_{j}\right]\right\}$. Applying $\left(*_{k}^{j-1}\right)$ to the first term, and $\left(*_{k+1}^{j}\right)$ to the second term, we obtain

$$
\left|f^{[k]}(\sigma(s))\right| \leq B_{j-1, n-k+1}^{(\alpha)} M_{k}^{\mathrm{c}}+\alpha \frac{M_{n-j}^{\mathrm{c}}}{M_{n-j+1}^{\mathrm{c}}} B_{j, n-k}^{(\alpha)} M_{k+1}^{\mathrm{c}} .
$$

Since $M^{\mathrm{c}}$ is $\log$-convex and $k \leq n-j$, we have $M_{n-j}^{\mathrm{c}} / M_{n-j+1}^{\mathrm{c}} \leq M_{k}^{\mathrm{c}} / M_{k+1}^{\mathrm{c}}$, hence

$$
\left|f^{[k]}(\sigma(s))\right| \leq M_{k}^{\mathrm{c}}\left(B_{j-1, n-k+1}^{(\alpha)}+\alpha B_{j, n-k}^{(\alpha)}\right)=B_{j, n-k+1}^{(\alpha)} M_{k}^{\mathrm{c}} .
$$

Thus $\left(*_{k}^{j}\right)$ holds, and both inductions may now proceed.

Next, by Lemma 4.6, there exists a constant $K>0$ such that, for all $u, v \in \mathbb{N}$ with $v>u$, we have $B_{u, v}<\alpha K$. Thus $|f(\sigma(t))| \leq K \alpha M_{0}$ for all $t \in[0, \Lambda(\sigma)]$. It follows that $f(\sigma(t))=0$ for all $t \in[0, \Lambda(\sigma)]$. In particular, $|f(z)|=0$ and hence $f(z)=0$. Since $z \in \Gamma^{*}$ was arbitrary, the above holds for all $z \in \Gamma^{*}$.

In the remainder of this paper we adopt the following convention. Let $X$ be a semirectifiable compact plane set, let $\mathcal{F}$ be an effective collection of paths in $X$, and let $f \in D_{\mathcal{F}}^{(\infty)}(X)$. If there exists $k \in \mathbb{N}_{0}$ such that $\left|f^{[k]}\right|_{X}=0$ then we regard this as a degenerate case where we still write

$$
\sum_{j=1}^{\infty}\left|f^{[j]}\right|_{X}^{-1 / j}=\infty
$$

Our first corollary will be used in the next section. 
Corollary 4.8. Let $\gamma$ be an admissible path in $\mathbb{C}$, let $\mathcal{F}$ be an effective collection of paths in $\gamma^{*}$, and let $f \in D_{\mathcal{F}}^{(\infty)}\left(\gamma^{*}\right)$. Suppose that

$$
\sum_{j=1}^{\infty}\left|f^{[j]}\right|^{-1 / j}=\infty
$$

If there exists $z_{0} \in \gamma^{*}$ such that $f^{[k]}\left(z_{0}\right)=0$ for all $k \in \mathbb{N}_{0}$, then $f$ is identically zero on $\gamma^{*}$.

Proof. We may assume that $\left|f^{[k]}\right|_{\gamma^{*}}>0$ for all $k \in \mathbb{N}_{0}$, as the degenerate case is easily dealt with using, for example, Lemma 4.5. For each $k \in \mathbb{N}_{0}$, set $M_{k}=\left|f^{[k]}\right|_{\gamma^{*}}$. By (4.8), $M=\left(M_{k}\right)_{k=0}^{\infty}$ satisfies (4.1), and certainly $\left|f^{[k]}\right|_{\gamma^{*}} \leq M_{k}$ for all $k \in \mathbb{N}_{0}$. Suppose that there exists $z_{0} \in \gamma^{*}$ such that $f^{[k]}\left(z_{0}\right)=0$ for all $k \in \mathbb{N}_{0}$. Then $f\left(\gamma^{*}\right) \subseteq\{0\}$ by Theorem 4.7 .

Our next corollary asserts the existence of an $\mathcal{F}$-quasianalytic algebra of the form $D_{\mathcal{F}}(X, M)$.

Corollary 4.9. Let $X$ be a semirectifiable compact plane set, let $\mathcal{F}$ be an effective collection of paths in $X$, and let $M=\left(M_{n}\right)_{n=0}^{\infty}$ be an algebra sequence which satisfies (4.1). Then $D_{\mathcal{F}}(X, M)$ is $\mathcal{F}$-quasianalytic.

Proof. Fix $f \in D_{\mathcal{F}}(X, M)$. Then there exists $N \in \mathbb{N}$ large enough so that $\left|f^{[k]}\right|_{X} \leq M_{k}$ for all $k \in \mathbb{N}_{0}$ with $k \geq N$. If there exists $k \in \mathbb{N}_{0}$ such that $\left|f^{[k]}\right|_{X}=0$, then 4.8 holds, so suppose that $\left|f^{[k]}\right|_{X}>0$ for all $k \in \mathbb{N}_{0}$. Then

$$
\sum_{j=1}^{\infty}\left|f^{[j]}\right|_{X}^{-1 / j} \geq \sum_{j=N}^{\infty}\left|f^{[j]}\right|_{X}^{-1 / j} \geq \sum_{j=N}^{\infty} M_{j}^{-1 / j}=\infty .
$$

Thus, in either case, $f$ satisfies (4.8). Hence, by Corollary 4.8, if there exist $\gamma \in \mathcal{F}$ and $z_{0} \in \gamma^{*}$ such that $f^{[k]}\left(z_{0}\right)=0$ for all $k \in \mathbb{N}_{0}$, then $f\left(\gamma^{*}\right) \subseteq\{0\}$. It follows that $D_{\mathcal{F}}(X, M)$ is $\mathcal{F}$-quasianalytic.

Since $D^{(\infty)}(X) \subseteq D_{\mathcal{F}}^{(\infty)}(X)$, Theorem 4.7 generalises [9, Theorem 1.11] to obtain the following.

Corollary 4.10. Let $M=\left(M_{n}\right)_{n=0}^{\infty}$ be a sequence of positive real numbers such that $\sum_{n=1}^{\infty} M_{n}^{-1 / n}=\infty$. Let $\gamma$ be an admissible path in $\mathbb{C}$. Suppose that $f \in D^{(\infty)}(X), f^{(k)}\left(\gamma^{-}\right)=0$ and $\left|f^{(k)}\right|_{X} \leq M_{k}$ for $k=0,1, \ldots$ Then $f$ is identically zero on $\gamma^{*}$.

We conclude this section with a note about $\mathcal{F}$-analyticity, as introduced in [4] (see also [5]). Let $X$ be a semirectifiable compact plane set, let $\mathcal{F}$ be an effective collection of paths in $X$, and let $f \in D_{\mathcal{F}}^{(\infty)}(X)$. We say that $f$ 
is $\mathcal{F}$-analytic if

$$
\limsup _{k \rightarrow \infty}\left(\frac{\left|f^{[k]}\right|}{k !}\right)^{1 / k}<\infty
$$

This is a generalisation of the term analytic applied in [15, 16], and is applied to find sufficient conditions for maps to induce homomorphisms between the algebras $D_{\mathcal{F}}(X, M)$. (Note that, in [14], the term analytic was used for those functions on $X$ which extend to be analytic on a neighbourhood of $X$. This condition is stronger than the one in [15, 16].)

Let $X$ be a semirectifiable compact plane set, let $\mathcal{F}$ be an effective collection of paths in $X$, and let $f \in D_{\mathcal{F}}^{(\infty)}(X)$. Using Theorem 4.7, we can show that if $f$ is $\mathcal{F}$-analytic then, for each $\gamma \in \mathcal{F}$ and $z \in \gamma^{*}$, there exist $r>0$ and an analytic (in the usual sense) function $g: \mathrm{B}(z, r) \rightarrow \mathbb{C}$ such that $g\left|\left(\gamma^{*} \cap \mathrm{B}(z, r)\right)=f\right|\left(\gamma^{*} \cap \mathrm{B}(z, r)\right)$. From this, it follows that in fact, for each $\gamma \in \mathcal{F}$, there exist an open neighbourhood $U$ of $\gamma^{*}$ and an analytic function $h: U \rightarrow \mathbb{C}$ such that $h\left|\gamma^{*}=f\right| \gamma^{*}$. We wish to thank Prof. J. K. Langley and Dr. D. A. Nicks for showing us how to prove the latter implication.

5. Trivial Jensen measures without regularity. We conclude the paper with an application of the results from the previous sections. We construct a locally connected compact plane set $X$ and an essential uniform algebra $A$ on $X$ such that $A$ does not admit any non-trivial Jensen measures but is not regular. This example will improve an earlier example of the first author [12].

We begin with the relevant definitions.

Definition 5.1. Let $X$ be a compact Hausdorff space, let $A$ be a uniform algebra on $X$, and let $\varphi$ be a character on $A$. A probability measure $\mu$ on $X$ is a Jensen measure for $\varphi$ (with respect to $A$ ) if

$$
\log |\varphi(f)| \leq \int_{X} \log |f| d \mu \quad(f \in A) .
$$

We say that $A$ is regular on $X$ if, for each closed set $E \subseteq X$ and each point $x \in X \backslash E$, there exists $f \in A$ such that $f(x)=0$ and $f(E) \subseteq\{0\}$. We say that $A$ is regular if the Gelfand transform of $A$ is regular on the character space $\Phi_{A}$ of $A$. We say that $A$ is essential if there exists no proper closed subset $E$ of $X$ such that $A$ contains every $f \in C(X)$ such that $f(y)=0$ for all $y \in E$.

In the above definition we adopt the convention that $\log 0=-\infty$. Let $X, A, \varphi$ be as above. It is standard that every Jensen measure for $\varphi$ is a representing measure for $\varphi$. Moreover, for each $\varphi \in \Phi_{A}$, there is a Jensen measure on $X$ for $\varphi$. Note that, for $x \in X$, the point mass measure $\delta_{x}$ is a Jensen measure for $\varepsilon_{x}$, where (here, and for the remainder of the section) 
$\varepsilon_{x}$ is the evaluation character at $x$ (with respect to $A$ ). We say that a Jensen measure $\mu$ on $X$ for $\varepsilon_{x}$ is trivial if $\mu=\delta_{x}$.

Let $X$ be a compact plane set. Let $R_{0}(X)$ denote the set of restrictions to $X$ of rational functions with no poles on $X$. Let $R(X)$ denote the uniform closure of $R_{0}(X)$ in $C(X)$. It is standard that $R_{0}(X)$ is an algebra and that $R(X)$ is a natural uniform algebra on $X$. For the remainder of this section, all Jensen measures will be with respect to $R(X)$ unless otherwise specified.

For further details on uniform algebras, Jensen measures, and related topics, see [3, 8, 22, 23, 28,

Let $x \in X$. Let $J_{x}$ denote the ideal in $R(X)$ of all functions which vanish on a neighbourhood of $x$. Let $M_{x}$ denote the ideal in $R(X)$ of all functions which vanish at $x$. Clearly $J_{x} \subseteq M_{x}$. We say that $x$ is a point of continuity (for $R(X)$ ) if, for all $y \in X \backslash\{x\}$, we have $J_{y} \nsubseteq M_{x}$. We say that $x$ is an $R$-point if, for all $y \in X \backslash\{x\}$, we have $J_{x} \nsubseteq M_{y}$. For further information see [12, 19 21]. (Note that in [19] points of continuity are referred to as regularity points of type one, and $R$-points are referred to as regularity points of type two.)

It is standard that $R(X)$ is regular if and only if every point of $X$ is a point of continuity, and this holds if and only if every point of $X$ is an $R$-point. It is also standard that if $x$ is a point of continuity then the only Jensen measure for $\varepsilon_{x}$ is the point mass measure.

Let $X$ be a topological space and let $E$ be a subset of $X$. We denote by $\operatorname{int}_{X}(E)$ the interior of $E$ with respect to $X$. In particular, if $E \subseteq \mathbb{C}$ then $\operatorname{int}_{\mathbb{C}}(E)$ coincides with the usual interior of $E$.

For the remainder of this section, we denote the set of non-negative real numbers by $\mathbb{R}^{+}$. Let $X$ be a metric space, let $x \in X$, and let $r>0$. We denote the open ball in $X$ with centre $x$ and radius $r$ by $\mathrm{B}_{X}(x, r)$, and the corresponding closed ball by $\overline{\mathrm{B}}_{X}(x, r)$.

In the special case where $X=\mathbb{C}$, for each $a \in \mathbb{C}$ and $r>0$, we write $\mathrm{B}(a, r)=\mathrm{B}_{\mathbb{C}}(a, r)$ and $\overline{\mathrm{B}}(a, r)=\overline{\mathrm{B}}_{\mathbb{C}}(a, r)$. For each $a \in \mathbb{C}$, we set $\mathrm{B}(a, 0)=\emptyset$ and $\overline{\mathrm{B}}(a, 0)=\{a\}$.

Lemma 5.2. Let $X, Y$ be compact plane sets with $Y \subseteq X$. Suppose $R(Y)$ is regular. Then each point $y \in \operatorname{int}_{X}(Y)$ is a point of continuity for $R(X)$.

Proof. Let $y \in \operatorname{int}_{X}(Y)$, and let $x \in X$ with $x \neq y$. Then there exists $r>0$ such that $\overline{\mathrm{B}}_{X}(y, r) \subseteq Y$. Choose $\delta \in(0, r / 3)$ such that $|y-x|>2 \delta$. Since $R(Y)$ is regular, it follows (from [13, Lemma 1.6], for example) that $R(Y \cap \overline{\mathrm{B}}(y, r))$ is regular. Set $E:=\overline{\mathrm{B}}_{X}(y, r) \backslash \mathrm{B}_{X}(y, \delta)$. Then there exists a function $g \in R\left(Y \cap \overline{\mathrm{B}}_{X}(y, r)\right)$ such that $f(y)=1$ and $f(x)=0$ for all $x \in E$. Let $f \in C(X)$ be given by $f(z)=g(z)$ for all $z \in X \cap \overline{\mathrm{B}}_{X}(y, r)$ and $f(z)=0$ for all $z \in X \backslash \overline{\mathrm{B}}_{X}(y, r)$. It follows from [24, Corollary II.10.3] that $f \in R(X)$ and clearly $f$ vanishes on a neighbourhood of $x$. Thus $J_{x} \nsubseteq M_{y}$ 
(where these are ideals in $R(X)$ ). It follows that $y$ is a point of continuity for $R(X)$.

The following can be found in [21, Lemma 3.1].

Lemma 5.3. Let $X$ be a compact plane set and let $x \in X$. Suppose that there exists a neighbourhood $U$ of $x$ in $X$ such that every point in $U \backslash\{x\}$ is a point of continuity. Then $x$ is an R-point.

As in [18] (see also [17), we define abstract Swiss cheeses as follows.

Definition 5.4. An abstract Swiss cheese is a sequence $\mathbf{A}=\left(\left(a_{n}, r_{n}\right)\right)_{n=0}^{\infty}$ of elements of $\mathbb{C} \times \mathbb{R}^{+}$. Let $\mathbf{A}=\left(\left(a_{n}, r_{n}\right)\right)_{n=0}^{\infty}$ be an abstract Swiss cheese. Set

$$
X_{\mathbf{A}}:=\overline{\mathrm{B}}\left(a_{0}, r_{0}\right) \backslash \bigcup_{n=1}^{\infty} \mathrm{B}\left(a_{n}, r_{n}\right),
$$

and set $\rho(\mathbf{A})=\sum_{n=1}^{\infty} r_{n}$. We say that $\mathbf{A}$ is classical if $\rho(\mathbf{A})<\infty, r_{0}>0$ and for all $k \in \mathbb{N}$ with $r_{k}>0$ the following hold:

(a) $\overline{\mathrm{B}}\left(a_{k}, r_{k}\right) \subseteq \mathrm{B}\left(a_{0}, r_{0}\right)$;

(b) whenever $\ell \in \mathbb{N}$ with $r_{\ell}>0$ and $\ell \neq k$, we have

$$
\overline{\mathrm{B}}\left(a_{k}, r_{k}\right) \cap \overline{\mathrm{B}}\left(a_{\ell}, r_{\ell}\right)=\emptyset \text {. }
$$

We say that a compact plane set $X$ is a Swiss cheese set if there exists an abstract Swiss cheese $\mathbf{A}$ such that $X=X_{\mathbf{A}}$. We say that a Swiss cheese set $X$ is classical if there exists a classical abstract Swiss cheese $\mathbf{A}$ with $X=X_{\mathbf{A}}$. If $X$ is a classical Swiss cheese set then $X$ is uniformly regular (see the proof of [10, Theorem 8.3]) and $R(X)$ is essential (see [3, p. 167] or [13. Theorem 1.8]). It follows that if $\mathbf{A}$ is classical then $X_{\mathbf{A}}$ is also connected and locally connected.

In [12, the first author gave an example of a non-classical Swiss cheese set $X$ such that $R(X)$ has no non-trivial Jensen measures, but such that $R(X)$ is not regular. We shall show that there is a classical Swiss cheese set with these properties; this is the content of the following theorem, which is the main theorem of this section.

TheOREM 5.5. There exists a classical abstract Swiss cheese $\mathbf{A}=\left(\left(a_{n}, r_{n}\right)\right)$ such that $R\left(X_{\mathbf{A}}\right)$ is not regular and does not admit any non-trivial Jensen measures.

Most of the remainder of this section is devoted to the proof of this theorem. We require some preliminary results. The following proposition is [21, Lemma 4.1].

Proposition 5.6. Let $X$ be a compact plane set, let $Y$ be a non-empty closed subset of $X$, and let $x \in Y$. Suppose that no bounded component 
of $\mathbb{C} \backslash Y$ is contained in $X$, and that there exists a non-trivial representing measure $\mu$ for $\varepsilon_{x}$ with respect to $R(X)$ such that $\operatorname{supp} \mu \subseteq Y$. Then $\mu$ is a non-trivial representing measure for $\varepsilon_{x}$ with respect to $R(Y)$, and $R(Y) \neq C(Y)$.

Note that, if $\operatorname{int}_{\mathbb{C}}(X)=\emptyset$ then the condition on bounded components of $\mathbb{C} \backslash Y$ is automatically satisfied.

Let $X$ be a compact plane set with $\operatorname{int}_{\mathbb{C}}(X)=\emptyset$, let $x \in X$, and let $\mu$ be a non-trivial representing measure for $\varepsilon_{x}$. Let $Y=\operatorname{supp} \mu \cup\{x\}$, where supp $\mu$ denotes the closed support of $\mu$. Then, by the above, we must have $R(Y) \neq C(Y)$. In particular, as noted in [21], $Y$ must have positive area. (See also the Hartogs-Rosenthal theorem [22, Corollary II.8.4].) Combining these observations with Proposition 5.6 gives the following corollary, which we use below.

Corollary 5.7. Let $X$ be a compact plane set with $\operatorname{int}_{\mathbb{C}}(X)=\emptyset$, let $E$ be a closed subset of $X$, and let $x \in E$. Suppose that $E$ has area 0 , that $\mu$ is a Jensen measure for $\varepsilon_{x}$ with respect to $R(X)$, and $\mu$ is supported on $E$. Then $\mu$ is trivial.

We also require the following lemma, which is a special case of [21, Lemma 2.1].

Lemma 5.8. Let $X$ be a compact plane set and let $x \in X$. Suppose that $\mu$ is a non-trivial Jensen measure for $x$, and let $F$ be the closed support of $\mu$. Then, for all $y \in F \backslash\{x\}$, we have $J_{y} \subseteq M_{x}$. Thus $x$ is not a point of continuity and no point of $F \backslash\{x\}$ is an R-point.

The following estimates on derivatives are standard. See for example [21, Lemma 4.4]. (This result also appears in [12] but with some typographical errors.)

LEMma 5.9. Let $\mathbf{A}=\left(\left(a_{n}, r_{n}\right)\right)_{n=1}^{\infty}$ be an abstract Swiss cheese, and let $z \in \mathbb{C}$. For each $n \in \mathbb{N}$, let $d_{n}$ denote the distance from $\mathrm{B}\left(a_{n}, r_{n}\right)$ to $z$. Let $d_{0}=r_{0}-\left|z-a_{0}\right|$. Suppose that $d_{n}>0$ for all $n \in \mathbb{N}_{0}$. Then $z \in X_{\mathbf{A}}$ and, for all $f \in R_{0}\left(X_{\mathbf{A}}\right)$ and $k \in \mathbb{N}_{0}$, we have

$$
\left|f^{(k)}(z)\right| \leq k ! \sum_{j=0}^{\infty} \frac{r_{j}}{d_{j}^{k+1}}|f|_{X_{\mathbf{A}}} .
$$

Our construction will use the following proposition, which is a combination of [18, Lemma 8.5] and, for example, [13, Example 2.9].

Proposition 5.10. Let $a \in \mathbb{C}, \lambda_{1} \geq 0, \lambda_{0}>\lambda_{1}$, and $\varepsilon>0$. Then there exists a classical abstract Swiss cheese $\mathbf{A}=\left(\left(a_{n}, r_{n}\right)\right)_{n=0}^{\infty}$ such that $a_{0}=a_{1}=a$, $r_{0}=\lambda_{0}$ and $r_{1}=\lambda_{1}$ such that $R\left(X_{\mathbf{A}}\right)$ is regular and $\sum_{n=2}^{\infty} r_{n}<\varepsilon$.

Note that, since $R\left(X_{\mathbf{A}}\right)$ is regular, we must have $\operatorname{int}_{\mathbb{C}}\left(X_{\mathbf{A}}\right)=\emptyset$. 
We now give the details of the construction.

Lemma 5.11. Let $0<r<1$ be given, let $C_{r}$ denote the circle of radius $r$ centred at 0 , and let $\varepsilon>0$. Then there exists a classical abstract Swiss cheese $\mathbf{A}=\left(\left(a_{n}, r_{n}\right)\right)$ such that:

(a) $\rho(\mathbf{A})<\varepsilon \operatorname{int}_{\mathbb{C}}\left(X_{\mathbf{A}}\right)=\emptyset$, and $C_{r} \subseteq X_{\mathbf{A}}$;

(b) there is a dense open subset $U$ of $X_{\mathbf{A}}$ such that $X_{\mathbf{A}} \backslash U$ has area zero and each point $z \in U$ is a point of continuity for $R\left(X_{\mathbf{A}}\right)$;

(c) for each $f \in R(X)$, we have

$$
f \mid C_{r} \in D^{(\infty)}\left(C_{r}\right) \quad \text { and } \quad \sum_{k=1}^{\infty}\left|f^{(k)}\right|_{C_{r}}^{-1 / k}=\infty .
$$

Proof. Our abstract Swiss cheese $\mathbf{A}$ will be obtained by combining a certain pair of sequences $\left(\mathbf{A}_{n}\right),\left(\mathbf{B}_{n}\right)$ of abstract Swiss cheeses in a suitable way. We first construct $\left(\mathbf{A}_{n}\right),\left(\mathbf{B}_{n}\right)$.

Choose a positive integer $n_{0}$ large enough so that $r+2^{1-n_{0}}<1$ and $r-2^{1-n_{0}}>0$. As in [21], choose a sequence $\left(\gamma_{n}\right)$ of positive real numbers such that, for each $n, k \in \mathbb{N}$, we have

$$
\gamma_{n} \leq \frac{\left(2^{1-n_{0}-n}\right)^{k+1}(\log (k+3))^{k}}{2^{k}},
$$

and such that $\sum_{n=1}^{\infty} \gamma_{n}<\varepsilon$. Thus, for each $k \in \mathbb{N}$,

$$
\sum_{n=1}^{\infty} \frac{\gamma_{n}}{\left(2^{1-n_{0}-n}\right)^{k+1}} \leq(\log (k+3))^{k} .
$$

Let $\mathbf{A}_{1}=\left(\left(a_{n}^{(1)}, r_{n}^{(1)}\right)\right)$ be the classical abstract Swiss cheese obtained from Proposition 5.10 with $\lambda_{0}=r-2^{1-n_{0}}, \lambda_{1}=0, a=0$ and $\varepsilon=\gamma_{1} / 2$. Let $\mathbf{B}_{1}=\left(\left(b_{n}^{(1)}, r_{n}^{(1)}\right)\right)$ be the classical abstract Swiss cheese obtained from Proposition 5.10 with $a=0, \lambda_{0}=1$ and $\lambda_{1}=r+2^{1-n_{0}}$ and $\varepsilon=\gamma_{1} / 2$.

For each $k \in \mathbb{N}$ with $n \geq 2$ : let $\mathbf{A}_{k}=\left(\left(a_{n}^{(k)}, r_{n}^{(k)}\right)\right)$ be the classical abstract Swiss cheese obtained from Proposition 5.10 applied with $\lambda_{0}=r-2^{2-n_{0}-k}$, $\lambda_{1}=r-2^{3-n_{0}-k}, a=0$ and $\varepsilon=\gamma_{k} / 2$; let $\mathbf{B}_{k}=\left(\left(b_{n}^{(k)}, r_{n}^{(k)}\right)\right)$ be the classical abstract Swiss cheese obtained from Proposition 5.10 applied with $a=0$, $\lambda_{0}=r+2^{3-n_{0}-k}$ and $\lambda_{1}=r+2^{2-n_{0}-k}$ and $\varepsilon=\gamma_{k} / 2$.

Let $a_{0}=0$ and $r_{0}=1$ and let $\left(\left(a_{n}, r_{n}\right)\right)_{n=1}^{\infty}$ be an enumeration (without repeats) of the set

$$
\bigcup_{\substack{m, n \in \mathbb{N} \\ n \geq 2}}\left\{\left(a_{n}^{(m)}, r_{n}^{(m)}\right),\left(b_{n}^{(m)}, s_{n}^{(m)}\right)\right\} .
$$

Then $\mathbf{A}=\left(\left(a_{n}, r_{n}\right)\right)_{n=0}^{\infty}$ is an abstract Swiss cheese with $C_{r} \subseteq X_{\mathbf{A}}$. It is not hard to see that $\mathbf{A}$ is classical and $\rho(\mathbf{A})<\varepsilon$. It is clear that $\operatorname{int}_{\mathbb{C}}\left(X_{\mathbf{A}}\right)=\emptyset$. 
Also, for each $n \in \mathbb{N}$, let

$$
E_{n}:=\partial \mathrm{B}\left(a_{0}^{(n)}, r_{0}^{(n)}\right) \cup \partial \mathrm{B}\left(a_{1}^{(n)}, r_{1}^{(n)}\right) \cup \partial \mathrm{B}\left(b_{0}^{(n)}, s_{0}^{(n)}\right) \cup \partial \mathrm{B}\left(b_{1}^{(n)}, s_{1}^{(n)}\right)
$$

(where $\partial S$ denotes the boundary of $S \subseteq \mathbb{C}$ ), and set $E:=C_{r} \cup \bigcup_{n=1}^{\infty} E_{n}$. Set $X:=X_{\mathbf{A}}$ and $U:=X \backslash E$. It is easy to see that $E$ is a closed set and that $U$ is a dense open subset of $X$. Moreover, $E$ has area zero.

We claim that each point $x \in U$ is a point of continuity for $R(X)$. Let $x \in U$. Then there exists a unique $n \in \mathbb{N}$ such that either $x \in X_{\mathbf{A}_{n}}$ or $x \in X_{\mathbf{B}_{n}}$. If $x \in X_{\mathbf{A}_{n}}$, set $Y=X_{\mathbf{A}_{n}}$, and if $x \in X_{\mathbf{B}_{n}}$ then set $Y=X_{\mathbf{B}_{n}}$. By our construction $R(Y)$ is regular and it is not hard to see that $x \in \operatorname{int}_{X}(Y)$. Thus, by Lemma 5.2, $x$ is a point of continuity for $R(X)$. This proves the claim.

It remains to show that (c) holds. We first consider functions in $R_{0}(X)$. Let $z \in C_{r}$ and let $f \in R_{0}(X)$. For each $n \in \mathbb{N}$, let $d_{n}$ be the distance from $\overline{\mathrm{B}}\left(a_{n}, r_{n}\right)$ to $C_{r}$, and let $d_{0}=1-r$. Since each $\mathbf{A}_{n}$ and each $\mathbf{B}_{n}$ are classical, and since $C_{r} \nsubseteq X_{\mathbf{A}_{n}}, X_{\mathbf{B}_{n}}$ for all $n \in \mathbb{N}$, it follows that $d_{n}>0$ for all $n \in \mathbb{N}_{0}$. By Lemma 5.9 , for each $k \in \mathbb{N}_{0}$, we have

$$
\left|f^{(k)}(z)\right| \leq k ! \sum_{j=0}^{\infty} \frac{r_{j}}{d_{j}^{k+1}}|f|_{X} .
$$

Fix $m \in \mathbb{N}$. Then there exists a unique $n \in \mathbb{N}$ such that there exists $\ell \in \mathbb{N}$ with $\left(a_{m}, r_{m}\right)=\left(a_{\ell}^{(n)}, r_{\ell}^{(n)}\right)$ or there exists $\ell \in \mathbb{N}$ with $\left(a_{m}, r_{m}\right)=\left(b_{\ell}^{(n)}, s_{\ell}^{(n)}\right)$. In either case, since $\mathbf{A}_{n}$ and $\mathbf{B}_{n}$ are classical, we have $d_{m}>2^{3-n_{0}-m}$. Thus

$$
k ! \sum_{j=0}^{\infty} \frac{r_{j}}{d_{j}^{k+1}}|f|_{X} \leq k !|f|_{X}\left(\frac{1}{d_{0}^{k+1}}+\sum_{j=1}^{\infty} \frac{\gamma_{j}}{\left(2^{1-n_{0}-j}\right)^{k+1}}\right)
$$

for each $k \in \mathbb{N}_{0}$. It follows that, for each $k \in \mathbb{N}_{0}$, we have

$$
\left|f^{(k)}\right|_{C_{r}} \leq k !|f|_{X}\left(\frac{1}{d_{0}^{k+1}}+(\log (k+3))^{k}\right) .
$$

From this we deduce that, for each $f \in R(X)$ (not necessarily in $R_{0}(X)$ ), we have $f \mid C_{r} \in D^{(\infty)}\left(C_{r}\right)$ and the same estimates hold. (One way to see this is to note that $C_{r}$ is uniformly regular, and apply [10, Theorem 5.6].)

Now let $f \in R(X)$. As in [21], choose $N \in \mathbb{N}$ large enough so that $(\log (k+3))^{k} \geq 1 / d_{0}^{k+1}$ for all $k \in \mathbb{N}$ with $k \geq N$. Then

$$
\sum_{j=1}^{\infty} \frac{1}{\left|f^{(j)}\right|_{C_{r}}^{1 / j}} \geq \sum_{j=N}^{\infty} \frac{1}{\left(2|f|_{X}\right)^{1 / j} j \log (j+3)}=\infty,
$$

and so (c) holds.

Proof of Theorem 5.5. Apply Lemma 5.11 with $r=1 / 2$ and $\varepsilon=1$ to obtain a classical abstract Swiss cheese $\mathbf{A}$ with properties (a)-(c) described 
in the statement of the lemma, and let $X:=X_{\mathbf{A}}$. Then, for each $f \in R(X)$, we have

$$
f \mid C_{r} \in D^{(\infty)}\left(C_{r}\right) \text { and } \quad \sum_{n=1}^{\infty}\left|f^{(n)}\right|_{C_{r}}^{-1 / n}=\infty .
$$

So, by Corollary 4.8 , if $f \in R(X)$ is such that $f^{(k)}(z)=0$ for some $z \in C_{r}$ and all $k \in \mathbb{N}_{0}$ then $f$ is identically zero on $C_{r}$. It follows that no point of $C_{r}$ can be a point of continuity for $R(X)$ and therefore $R(X)$ is not regular. It remains to see that $R(X)$ has no non-trivial Jensen measures on $X$. By Lemma 5.11(b) there is a dense open subset $U$ of $X$ for which every point $z \in U$ is a point of continuity for $R(X)$ and such that $X \backslash U$ has area 0. Set $E:=X \backslash U$. By Lemma 5.3 every point of $U$ is also an $R$-point. Let $x \in C_{r}$ and let $\mu$ be a Jensen measure for $\varepsilon_{x}$. Then since $x \notin U$, by Lemma 5.8 , $\operatorname{supp} \mu \cap U=\emptyset$ and so supp $\mu \subseteq E$. Since $\operatorname{int}_{\mathbb{C}}(X)=\emptyset$ and the area of $E$ is 0 , it follows from Corollary 5.7 that $\mu$ must be trivial. This completes the proof of Theorem 5.5 .

It is also possible to show that $R\left(X_{\mathbf{A}}\right)$ admits no non-trivial Jensen measures by appealing to the theory of Jensen interior. (See, for example, [24, p. 319].) This is the approach used in [12].

Our final corollary follows immediately from Theorem 5.5 .

COROLlary 5.12. There exists a locally connected compact plane set $X$ such that $R(X)$ is essential, non-trivial and non-regular and yet $R(X)$ admits no non-trivial Jensen measures.

We conclude with some open questions.

Question 5.13. Is the uniform algebra $R\left(X_{\mathbf{A}}\right)$ constructed in Theorem 5.5 necessarily antisymmetric? If not, can the construction be modified to yield an example which has the properties in that theorem and is also antisymmetric?

Question 5.14. Let $A$ be a uniform algebra on a compact Hausdorff space $X$, and let $M_{i}(i \in I)$ be the maximal $A$-antisymmetric subsets of $X$.

(a) Suppose that $A \mid M_{i}$ is regular on $M_{i}$ for all $i \in I$. Must $A$ be regular on $X$ ? What if we assume the stronger condition that $A \mid M_{i}$ is regular (so natural and regular on $M_{i}$ ) for all $i \in I$ ?

(b) What is the answer to (a) in the special case where $X$ is a compact plane set and $A=R(X)$ ?

Acknowledgments. We would like to thank the referee for helpful comments. The second author was supported by EPSRC grant number $\mathrm{EP} / \mathrm{L} 50502 \mathrm{X} / 1$. 


\section{References}

[1] T. M. Apostol, Mathematical Analysis, 2nd ed., Addison-Wesley, Reading, MA, 1974.

[2] W. J. Bland and J. F. Feinstein, Completions of normed algebras of differentiable functions, Studia Math. 170 (2005), 89-111.

[3] A. Browder, Introduction to Function Algebras, Benjamin, New York, 1969.

[4] T. Chaobankoh, Endomorphisms of Banach function algebras, PhD thesis, Univ. of Nottingham, 2012.

[5] T. Chaobankoh, J. F. Feinstein, and S. Morley, The chain rule for $\mathcal{F}$-differentiation, Irish Math. Soc. Bull. 77 (2016), 19-34.

[6] P. J. Cohen, A simple proof of the Denjoy-Carleman theorem, Amer. Math. Monthly 75 (1968), 26-31.

[7] J. B. Conway, Functions of One Complex Variable, Grad. Texts in Math. 11, Springer, New York, 2nd ed., 1978.

[8] H. G. Dales, Banach Algebras and Automatic Continuity, London Math. Soc. Monogr. (N.S.) 24, Clarendon Press, New York, 2000.

[9] H. G. Dales and A. M. Davie, Quasianalytic Banach function algebras, J. Funct. Anal. 13 (1973), 28-50.

[10] H. G. Dales and J. F. Feinstein, Normed algebras of differentiable functions on compact plane sets, Indian J. Pure Appl. Math. 41 (2010), 153-187.

[11] H. Federer, Geometric Measure Theory, Grundlehren Math. Wiss. 153, Springer, New York, 1969.

[12] J. F. Feinstein, Trivial Jensen measures without regularity, Studia Math. 148 (2001), $67-74$.

[13] J. F. Feinstein and M. J. Heath, Swiss cheeses, rational approximation and universal plane curves, Studia Math. 196 (2010), 289-306.

[14] J. F. Feinstein and H. Kamowitz, Endomorphisms of Banach algebras of infinitely differentiable functions on compact plane sets, J. Funct. Anal. 173 (2000), 61-73.

[15] J. F. Feinstein and H. Kamowitz, Compact endomorphisms of Banach algebras of infinitely differentiable functions, J. London Math. Soc. 69 (2004), 489-502.

[16] J. F. Feinstein and H. Kamowitz, Compact homomorphisms between Dales-Davie algebras, in: Banach Algebras and Their Applications, Contemp. Math. 363, Amer. Math. Soc., Providence, RI, 2004, 81-87.

[17] J. F. Feinstein, S. Morley, and H. Yang, Swiss cheeses and their applications, in: Function Spaces in Analysis, Contemp. Math. 645, Amer. Math. Soc., Providence, RI, 2015, 99-118.

[18] J. F. Feinstein, S. Morley, and H. Yang, Abstract Swiss cheese space and classicalisation of Swiss cheeses, J. Math. Anal. Appl. 438 (2016), 119-141.

[19] J. Feinstein and R. Mortini, Partial regularity and t-analytic sets for Banach function algebras, Math. Z. 271 (2012), 139-155.

[20] J. F. Feinstein and D. W. B. Somerset, Non-regularity for Banach function algebras, Studia Math. 141 (2000), 53-68.

[21] J. F. Feinstein and H. Yang, Regularity points and Jensen measures for $R(X)$, Studia Math. 235 (2016), 1-15.

[22] T. W. Gamelin, Uniform Algebras, Prentice-Hall, Englewood Cliffs, NJ, 1969.

[23] T. W. Gamelin, Uniform Algebras and Jensen Measures, London Math. Soc. Lecture Note Ser. 32, Cambridge Univ. Press, Cambridge, 1978.

[24] T. W. Gamelin and T. J. Lyons, Jensen measures for $R(K)$, J. London Math. Soc. (2) 27 (1983), 317-330. 
[25] P. Koosis, The Logarithmic Integral. I, Cambridge Stud. Adv. Math. 12, Cambridge Univ. Press, Cambridge, 1998.

[26] S. Mandelbrojt, Séries adhérentes, régularisation des suites, applications, GauthierVillars, Paris, 1952.

[27] W. Rudin, Real and Complex Analysis, 3rd ed., McGraw-Hill, New York, 1987.

[28] E. L. Stout, The Theory of Uniform Algebras, Bogden \& Quigley, Tarrytown-onHudson, NY, 1971.

J. F. Feinstein, S. Morley

School of Mathematical Sciences

The University of Nottingham

University Park

Nottingham, NG7 2RD, UK

E-mail: joel.feinstein@nottingham.ac.uk pmxsm9@nottingham.ac.uk 